\title{
Probing the low and high density nuclear matter by hadron scat- tering
}

\author{
S.M. Eliseev ${ }^{1}$, O.S. Kosmachev ${ }^{1}$ \\ ${ }^{1}$ Joint Institute for Nuclear Research, 141980, Dubna, Moscow Region, Russia
}

\begin{abstract}
The subject of this report is connected with the widely discussed manifestation of in-medium effect in nuclear collisions. At present, the medium modification of hadron properties in the normal and in a dense nuclear matter is one of the fundamental questions of strong interaction physics. A new Glauber Monte Carlo model for hadron-nuclei interaction at intermediate energy is proposed. We utilized the principal assumptions as in the approaches of other authors describing nuclear collisions at high energy in the framework of the models without QGP. Yet, a number of new ingredients (noneikonal corrections, correlations of nucleons, in the nuclei, the nuclear Fermi motion, etc.) are introduced.
\end{abstract}

\section{Introduction}

Whether or not hadrons really change their properties in a nuclear medium, has been one of the longstanding problems in nuclear physics [1-4]. One of the essential assumption of long-familiar nuclear physics is that the protons and neutrons do not significantly change their properties and structure when they are in a nucleus.

Relativistic heavy-ion collisions are considered to be a favorable situation for the study of a large number of different phenomena which are expected to identify new properties of nuclear matter and nuclear interactions predicted by QCD. From QCD we know that at a sufficiently density, which may be obtained in high energy nucleus-nucleus collisions, color screening will lead to quark deconfinement. One of the most important subject discussed at present, are the difficulties related to the long distances in QCD. However, nucleons can significantly change their properties even in the ground state of nuclei $[3,4]$. As it is seen from figure 1, the structure of nucleons is modified inside the nuclear medium (in-medium effect) at density $\rho \geq 0.5 \rho_{0}, \rho_{0}$ is normal nuclear matter density. Inclusion of such unconventional EMC-type effects draw greater attention in nuclear physics [1,2].

The renormalization of mass in the nuclear medium should be taken as a signature of partial restoration of chiral symmetry in ground state nuclei, even below the critical temperature and density. Such observations can direct to a some bridge between the investigations at intermediate and high energy. "This would represent a new paradigm for nuclear physics and help to build a bridge between QCD and nuclei "[1,2].

In the last years, a lot of theoretical and experimental work has been devoted to the search of signals of such anomalous properties of hadrons in the nuclear environment in-medium effects [1]. 
Hadron modification has been one of the most debated topics in nuclear and particle physics conferences and attracted a great attention of scientists in different branches of particle, nuclear physics and astrophysics [5, 6].
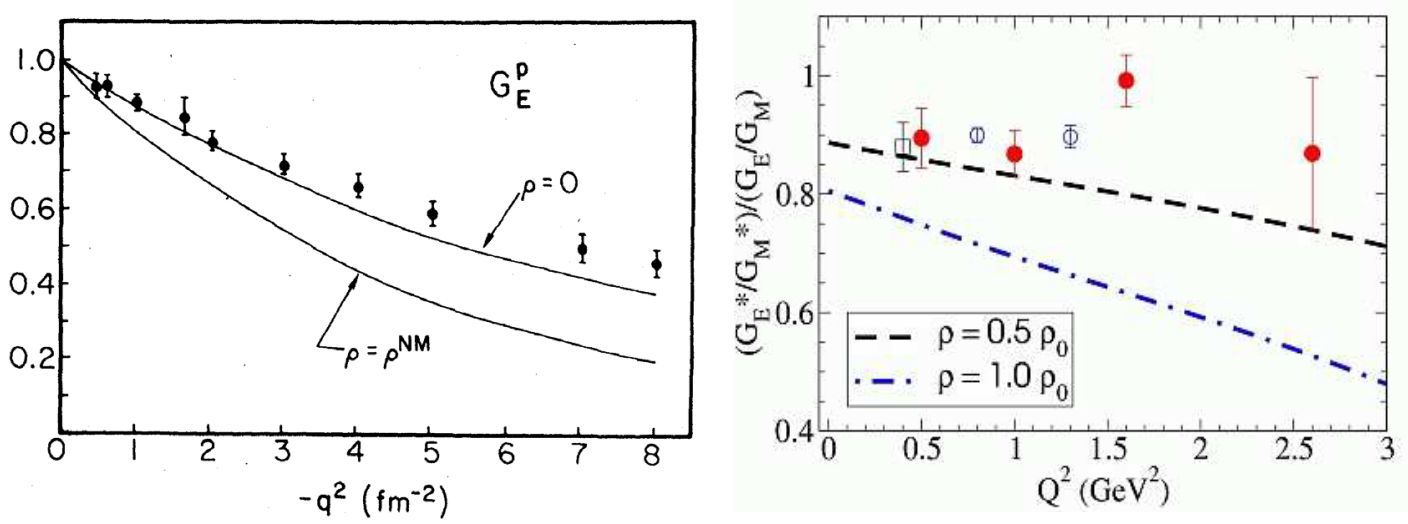

Figure 1. Left: calculated values of $G_{E}^{p}\left(q^{2}, \rho / \rho^{N M}\right)$ as a function of $-q^{2}$ for $\rho / \rho^{N M}=0$ (nucleon in vacuum) and for $\rho / \rho^{N M}=1$ (nucleon in nuclear matter). Figure is taken from[4], figure 5. Right: double ratio for the proton electric/magnetic form factors in ${ }^{4} \mathrm{He}$, normalized by the ratio in the vacuum versus the momentum transfer squared, $\mathrm{Q}^{2}$. Data are from Mainz (square data point) and Jefferson Lab (circular data points). Predictions are given for normal nuclear matter density $\left(\rho=1.0 \rho_{0}\right)$ and half normal nuclear density $\left(\rho=0.5 \rho_{0}\right)$. Note the $10 \%$ reduction for $\rho=0.5 \rho_{0}$ and the $20 \%$ reduction for $\rho=1.0 \rho_{0}$ at $\mathbf{Q}^{2}=0$. Figure is taken from [3], figure 14 .

\section{Glauber model}

Presently, partly due to our lack of knowledge about the details of the confined mechanism, we are far away from a possibility to resolve the problem of in-medium effects from the "first principles". Every attempt to extract some information concerning these effects from data will have (in part) the phenomenology.

Although the Glauber theory [7] was developed initially for high-energy projectiles, yet it is also employed at medium energies successfully $[8,9]$.

Glauber model became quite standard in the theoretical physics community, for details we refer to [10] and references therein. It is used in various theoretical models where it is essential to calculate the propagation of a particle through the medium. On the basis of this model several experimental data were interpreted, candidates for the signals of a phenomenon beyond the standard scenario: $\mathrm{J} / \Psi$ suppression, the phenomenon of color transparency, modeling in high energy nuclear collisions [10]. Therefore it is very tempting to use the same theoretical approach to recognize whether experiment on $\mathrm{K}^{+}$-nuclei provides us with a hint for some new physics.

Kaons play a unique role in strong interaction physics. To examine the nuclear interior, the $\mathrm{K}^{+}-$ meson is regarded as a unique probe due to its long mean free path in the nuclear matter. Among all the hadrons, this makes the $\mathrm{K}^{+}$the weakest strongly interacting particle. This also means that $\mathrm{K}^{+}$can penetrate into the interior of a nucleus, thus probing the nucleus in a region where the density is high $[11,12]$. 
According to the Glauber approximation the amplitude of a projectile-target elastic scattering may be written in the general form:

$$
f(\vec{q})=\frac{i k}{2 \pi} \int e^{i \vec{q} \cdot \vec{b}} \Gamma(\vec{b}) d \vec{b}, \text { and } \Gamma(\vec{b})=1-e^{i \chi(\vec{b})}=\frac{1}{2 \pi i k} \int e^{-i \vec{q} \cdot \vec{b}} f(\vec{q}) d \vec{q},
$$

where $\vec{b}$ is the impact parameter, $\chi$ is the corresponding phase shift function, and $f(\vec{q})$ is the elastic scattering amplitude of kaon-nucleon interaction.

Further, given the corresponding projectile-target nucleon amplitudes $f(q)$, one can express the above projectile-target nucleus amplitude in the parameter-free way. For projectile-nucleus scattering equation can be cast into the form:

$$
F(\vec{Q})=\frac{i k}{2 \pi} \int e^{i \vec{Q} \cdot \vec{b}}<\left[1-e^{i \chi\left(\vec{b}, \vec{s}_{1}, \cdots, \vec{s}_{A}\right)}\right]>d \vec{b}
$$

where $\overrightarrow{s_{j}}$ is a component of the radius-vector $\overrightarrow{r_{j}}$ of the $j^{t h}$ target-nucleon in the direction perpendicular to the incident momentum $\vec{k}$, while the brackets $<>$ denote the target ground-state average.

We used corrections to the Glauber's approximation which have been obtained by Wallace [13]:

$$
\chi_{j}(b)=\sum_{n=0}^{j} \chi^{(n)}(b), \chi^{(n)}(b)=-\frac{\mu^{n+1}}{k(n+1) !}\left(\frac{b}{k^{2}} \frac{\partial}{\partial b}-\frac{\partial}{\partial k} \frac{1}{k}\right)^{n} \int_{-\infty}^{\infty} V^{n+1}(r) d z, V(r)=\frac{2 \pi i}{\mu} f(0) \rho(r),
$$

where $\mu$ is a reduced mass, and $k$ is the momentum in the c.m.s. $(\hbar=c=1)$. The zeroth-order term in the above expansion represents the Glauber eikonal phase shift function, whereas higher-order terms, noneikonal effects. In this work, we truncate the tedious expansion of (3) and include, into our calculations, noneikonal corrections up to $3^{\text {rd }}$ order. Then, the nuclear phase shift function can be written as follows,

$$
\chi(b)=\chi_{0}(b)+\chi_{N E}(b), \quad \chi_{0}(b)=\frac{2 \pi}{k} \int_{-\infty}^{\infty} f(0) \rho(r) d z=-\frac{\mu}{k} \int_{-\infty}^{\infty} V(r) d z
$$

where $f(0)$ is the elementary forward scattering amplitude, $\rho(r)$ is the corresponding nuclear density. Also, one needs to point to the fact that the ${ }^{28} \mathrm{Si}$ nucleus is significantly clustered. Details of the nuclear structure is important for calculating differential cross sections [9]. In the report, we calculate only the total cross sections (see figure 2), and in this case it is necessary to know only the average density distribution of the nucleus [7,8].

The parameters of kaon-nucleon amplitudes was taken from the partial wave analysis of $\mathrm{K}^{+}$nucleon scattering (for details we refer to [9] and references therein). Having thus the amplitudes $f(I)$ for isospins $I=0$ and $I=1$ we can readily find the $\mathrm{K}^{+}$-proton amplitude as well as $\mathrm{K}^{+}$-neutron amplitude

$$
f\left(K^{+} p \rightarrow K^{+} p\right)=f(I=1), \quad f\left(K^{+} n \rightarrow K^{+} n\right)=\frac{1}{2}[f(I=0)+f(I=1)] .
$$

For $\mathrm{N}=\mathrm{Z}$ nuclei we used the average $\mathrm{K}^{+}-\mathrm{N}$ amplitude

$$
f\left(K^{+} N\right)=\frac{1}{2}\left[f\left(K^{+} p\right)+f\left(K^{+} n\right)\right] .
$$


Further, given the corresponding projectile-target nucleon amplitudes $f(q)$, one can express the above projectile-target nucleus amplitude in the parameter-free way. It is then a straightforward matter to determine the total cross section for the case of $\mathrm{K}^{+}$- target nucleus scattering according to the optical theorem. The total and reaction cross sections may then be approximated by

$$
\sigma_{t}=4 \pi \int_{0}^{\infty} \operatorname{Re}\left[1-e^{i \chi(b)}\right] b d b, \quad \sigma_{r}=2 \pi \int_{0}^{\infty}\left[1-e^{-2 \operatorname{Im} \chi(b)}\right] b d b,
$$

where $\chi(b)$ is the nuclear phase shift function.

The detailed Fermi motion effect (using Monte Carlo simulation) are applied in the Glauber approach for nuclear collisions. In the present paper we consider a Gaussian parameterization of the momentum distribution of nucleons in nucleus used in analysis of high energy heavy-ion collisions and EMC effect [15]:

$$
\rho(p)=N\left(e^{-p^{2} / p_{0}^{2}}+\epsilon_{0} e^{-p^{2} / q_{0}^{2}}\right)
$$

where $p_{0}=\sqrt{2 / 5} k_{F}, \quad \epsilon_{0}=0.03, \quad q_{0}=\sqrt{3} p_{0}, k_{F}=221 \mathrm{MeV} / \mathrm{c}$ and $N$ is a normalization constant.

\section{Results and discussion}

In figure 2 we show the dependence of the calculated and experimental cross sections of $\mathrm{K}^{+}-{ }^{28} \mathrm{Si}$ interactions versus kaon momentum. We can see that there is a universal discrepancy between the theoretical models and data for particles $\left(\mathrm{K}^{+}\right.$-mesons) interacting with ${ }^{28} \mathrm{Si}$ nuclei. In addition, optical potential models fail to describe the experimental data on $\mathrm{K}^{+}$-nuclei interaction [12]. In comparison with previous works $[12,16-19]$, our approach improves the agreement between experimental data and theory. In addition, it is worth to emphasize that our results have been obtained without fitting any new parameters. Furthermore, $\mathrm{K}^{+}$-meson probes the nucleus in a region of high nuclear density $[12,16-19]$. At the same time, Glauber model gives an adequate description of collisions of proton with targets in the case of peripheral interactions, i.e. in the domain of low nuclear density [9].
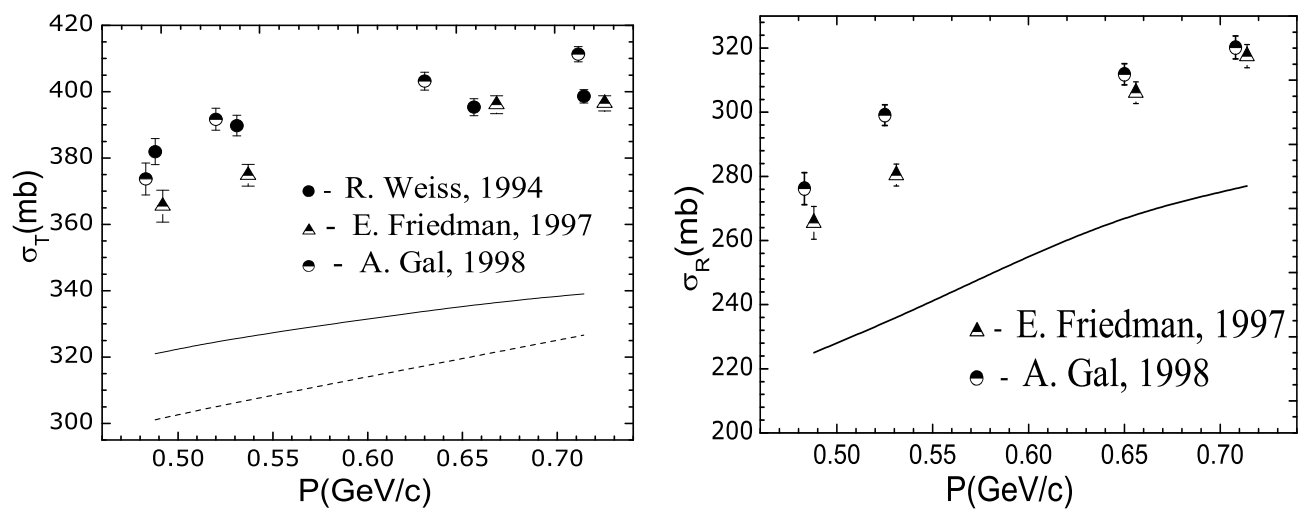

Figure 2. The calculated and experimental [16-18] total $\left(\sigma_{T}\right)$ and reaction $\left(\sigma_{R}\right)$ cross sections for $\mathrm{K}^{+}-$meson interaction with ${ }^{28} \mathrm{Si}$ nuclei versus kaon momentum. The solid lines denote the prediction from our model. The dotted line demonstrates the results from the models used to calculate kaon-nucleus scattering: the covariant momentum-space optical potential model and the eikonal model (see table II, page 862 from [19]). 
It is interesting to note - in both cases (electron-nucleon scattering and kaon-nucleus scattering) in-medium effect appears at dense nuclear matter, see figure 1 and figure 2. Comparison of the experimental data with the results of our calculations clearly demonstrates that the deeply penetrating hadron $\left(\mathrm{K}^{+}-\right.$meson) can "see" in the nucleus some "exotics" (beyond habitual mechanism of nuclear reactions). In our opinion the differences between models and data are more than just an artifact of the phenomenological models and that the introduction of some new in-medium effects is really required (see also [1-3, 5, 6]). In conclusion, investigation at intermediate energy of such type "constitutes an important step for the future studies of baryons in high density matter with a strong magnetic field such as in a neutron star (or a magnetar), and in heavy ion collision experiment" [1-3].

\section{References}

[1] A.W.Thomas, EPJ Web Conf. 123, 01003 (2016)

[2] C. Cloet, Wolfgang Bentz, and Anthony W. Thomas, Phys. Rev. Lett. 116, 032701 (2016)

[3] G. Ramalho, K. Tsushima, and A.W. Thomas, J. Phys. G 40, 015102 (2013)

[4] L. S. Celenza et al., Phys. Rev. C 31, 232 (1985)

[5] D. N. Voskresensky, D. Blaschke, Eur. Phys. J. A 52, 67 (2016)

[6] Wei-Zhou Jiang, Bao-An Li, F. J. Fattoyev, Eur. Phys. J. A 51, 119 (2015)

[7] R.G. Glauber, "Lectures in Theoretical Physics", edited by W.E. Brittin and L.G. Dunham (Interscience, New York, 1959), vol. 1, p. 315

[8] R.J. Glauber, G. Matthiae, Nucl. Phys. B 21, 135 (1970)

[9] R.H. Bassel and C. Wilkin. Phys. Rev. 174, 1179 (1968) and references therein

[10] Michael L. Miller et al., Ann. Rev. Nucl. Part. Sci. 57, (2007)

[11] G.E. Brown, C.B. Dover, P.B. Siegel, and W.Weise, Phys. Rev. Lett. 60, 2723 (1988)

[12] E. Friedman, A. Gal, Phys. Rept. 452, 89 (2007)

[13] S.J. Wallace, Ann. Phys. (N.Y.) 78, 190 (1973)

[14] J.S. Hyslop, R.A. Arndt, L.D. Roper, R.L. Workman, Phys. Rev. D 46, 961 (1992)

[15] Fujita T. and Hüfner J., Nucl. Phys. A 343, 493 (1980)

[16] R. Weiss et al., Phys. Rev. C 49, 2569 (1994)

[17] E. Friedman et al., Phys. Rev. C 55, 1304 (1997)

[18] A. Gal, Nucl. Phys. A 639, 485c (1998)

[19] M.F. Jiang, D.J. Ernst and C.M. Chen, Phys. Rev. C 51, 857 (1995) 